\title{
Docosenoic Acid n-9
}

National Cancer Institute

\section{Source}

National Cancer Institute. Docosenoic Acid n-9. NCI Thesaurus. Code C68353.

A monounsaturated very long-chain fatty acid with a 22-carbon backbone and a single double bond originating from the 9th position from the methyl end. 